Check for updates

Cite this: New J. Chem., 2018, 42, 16384

DOI: $10.1039 / c 8 n j 90083 c$

rsc.li/njc

\section{Correction: Naphthalene flanked diketopyrrolopyrrole based organic semiconductors for high performance organic field effect transistors}

\author{
Qian Liu, ${ }^{a}$ Huabin Sun, ${ }^{\mathrm{b}}$ Chula Blaikie, ${ }^{a}$ Chiara Caporale, ${ }^{c}$ Sergei Manzhos, ${ }^{d}$ \\ Krishna Feron, ${ }^{\text {ef }}$ Jennifer M. MacLeod, ${ }^{a}$ Massimiliano Massi, ${ }^{c}$ Steven E. Bottle, ${ }^{a}$ \\ John Bell, ${ }^{a}$ Yong-Young Noh ${ }^{\mathrm{b}}$ and Prashant Sonar*a
}

Correction for 'Naphthalene flanked diketopyrrolopyrrole based organic semiconductors for high performance organic field effect transistors' by Qian Liu et al., New J. Chem., 2018, 42, 12374-12385.

The authors would like to correct the Acknowledgements section. The Acknowledgements section should read:

Qian Liu is thankful to QUT for offering here QUTPRA scholarship to conduct his research. P. S. is thankful to QUT for the financial support from the Australian Research Council (ARC) for the Future Fellowship (FT130101337) and QUT core funding (QUT/322120-0301/07). S. M. is supported by the Ministry of Education of Singapore. Some of the data reported in this paper were obtained at the Central Analytical Research Facility (CARF) operated by the Institute for Future Environments (QUT). Access to CARF is supported by generous funding from the Science and Engineering Faculty (QUT). This study was supported by the Center for Advanced Soft-Electronics (2013M3A6A5073183) through the NRF grant funded by the Korean government.

The Royal Society of Chemistry apologises for these errors and any consequent inconvenience to authors and readers.

\footnotetext{
${ }^{a}$ School of Chemistry, Physics and Mechanical Engineering (CPME), Queensland University of Technology (QUT), Brisbane, QLD 400o, Australia.

E-mail: sonar.prashant@qut.edu.au

${ }^{b}$ Department of Energy \& Materials Engineering, Dongguk University, Seoul 04620, Republic of Korea. E-mail: yynoh@hotmail.com

${ }^{c}$ Department of Chemistry, Curtin University, GPO Box U1987, Perth 6845, Western Australia, Australia

${ }^{d}$ Department of Mechanical Engineering, Faculty of Engineering, National University of Singapore Block EA \#07-08, 9 Engineering Drive 1 117576, Singapore

${ }^{e}$ CSIRO Energy Centre, 10 Murray Dwyer Circuit, Mayfield West, NSW 2304, Australia

${ }^{f}$ Centre for Organic Electronics, University of Newcastle, Callaghan, NSW 2308, Australia
} 